\title{
A precise technique for the measurement of acoustic cavitation thresholds and some preliminary results
}

R. A. Roy, A. A. Atchley, L. A. Crum, J. B. Fowlkes, and J. J. Reidy

Citation: The Journal of the Acoustical Society of America 78, 1799 (1985); doi: 10.1121/1.392767

View online: https://doi.org/10.1121/1.392767

View Table of Contents: https://asa.scitation.org/toc/jas/78/5

Published by the Acoustical Society of America

\section{ARTICLES YOU MAY BE INTERESTED IN}

An acoustic backscattering technique for the detection of transient cavitation produced by microsecond pulses of ultrasound

The Journal of the Acoustical Society of America 87, 2451 (1990); https://doi.org/10.1121/1.399091

Thresholds for transient cavitation produced by pulsed ultrasound in a controlled nuclei environment

The Journal of the Acoustical Society of America 88, 2059 (1990); https://doi.org/10.1121/1.400102

Sonoluminescence and bubble dynamics for a single, stable, cavitation bubble

The Journal of the Acoustical Society of America 91, 3166 (1992); https://doi.org/10.1121/1.402855

The Role of Impurities in Cavitation-Threshold Determination

The Journal of the Acoustical Society of America 48, 1179 (1970); https://doi.org/10.1121/1.1912258

Cavitation dynamics. I. A mathematical formulation

The Journal of the Acoustical Society of America 57, 1379 (1975); https://doi.org/10.1121/1.380624

Acoustic cavitation prediction

The Journal of the Acoustical Society of America 69, 1624 (1981); https://doi.org/10.1121/1.385939 


\title{
A precise technique for the measurement of acoustic cavitation thresholds and some preliminary results
}

\author{
R. A. Roy, A. A. Atchley, L. A. Crum, J. B. Fowlkes, and J. J. Reidy \\ Department of Physics, University of Mississippi, Oxford, Mississippi 38677
}

(Received 7 September 1984; accepted for publication 30 July 1985)

\begin{abstract}
A description is given of a precise technique for measuring the threshold for acoustic cavitation inception. The system, which is automated so as to remove operator involvement, utilizes a slow ramping of the acoustic pressure amplitude until cavitation occurs. The detection criterion is the generation of a sufficiently intense sonoluminescent signal. Measurements made in filtered water show a well-defined, reproducible, and stable cavitation threshold. Measurements of the dependence of the threshold on filter size, on time, and on the concentration of dissolved ions for various salts are also presented. Many of these results appear anomalous.
\end{abstract}

PACS numbers: 43.25. Yw, 43.25.Zx, 43.30.Nb

\section{INTRODUCTION}

A remarkable property of water is its inability to sustain a tensile stress for even short periods of time. Laboratory measurements of the tensile strength of water have yielded values (e.g., 5 bars) that are significantly less than predictions based on homogenous nucleation theory ( $\approx 1000$ bars). The reduced strength is attributed to the existence of stable gas pockets (nuclei) that serve as preferential sites for liquid rupture. Since free gas bubbles are inherently unstable due to surface tension pressure, it has been necessary to formulate mechanisms ${ }^{1-4}$ that retard the dissolution of these gas pockets.

Whatever the nature of the nuclei, which is a controversial issue discussed at length elsewhere, ${ }^{5-8}$ we assume that they exist and are the sites of cavitation activity. For a degassed, acoustically stressed liquid, these nuclei may form rapidly growing vapor cavities which subsequently collapse violently during the ensuing positive pressure cycle. Such behavior, which is termed "transient acoustic cavitation," results in temperatures and pressures that are estimated to reach kilokelvins and kilobars for spherically symmetrical collapses. This potential for damage is of considerable importance to those who employ sound fields in fluid media.

The occurrence of cavitation is vitally dependent on the nature of the nuclei that initiate it; thus any information that provides a better understanding of cavitation nucleation is valuable. All the existing stabilization models are intimately connected with the composition of the liquid. The "crevice model" of Harvey ${ }^{1}$ and others ${ }^{9-11}$ assumes that gas is entrained in cracks on the surface of hydrophobic dirt particles or on the walls of a container. Akulichev ${ }^{2}$ has suggested that hydrophobic ions may collect on or near the surface of free bubbles and thus promote stabilization. According to Yount, ${ }^{4}$ free bubbles are stabilized by tough, surface active organic skins of varying permeability. Clearly, a systematic study of cavitation nuclei requires an apparatus that exercises adequate control over the physical and chemical properties of the liquid. In this paper, we present such an apparatus, describe a technique for the generation and detection of transient acoustic cavities, and report preliminary data that demonstrate the utility of this system.

\section{A. Definition of terms}

In this experiment, water is dynamically stressed by a spherically symmetrical stationary acoustic field (61.725 kHz). The minimum acoustic pressure amplitude necessary to initiate "cavitation" determines, in a general sense, the cavitation threshold. The choice of the detection criterion that establishes the presence of cavitation depends on the type of cavitation activity one wishes to monitor. Observers have relied on the appearance of bubbles, ${ }^{12}$ the presence of subharmonics ${ }^{13}$ (primarily nonlinear oscillations of stable gas bubbles), audible clicks and pops ${ }^{14}$ (shock waves generated by a transient cavity), as well as a variety of chemical and physical mechanisms. For the time being, we choose to consider only transient cavitation, where the collapse temperature and pressure is sufficient to induce light emission from the interior of the bubble. This emission (sonoluminescence) serves as an indication of cavitation activity. We therefore determine the transient cavitation threshold by slowly ramping the acoustic pressure amplitude until sonoluminescence occurs. Unless otherwise stated, all subsequent references to cavitation and cavitation thresholds refer to transient bubble activity.

\section{EXPERIMENTAL APPARATUS AND PROCEDURE}

A large number of experimental systems have been used to generate and detect acoustic cavitation, many of which are described in a review by Blake. ${ }^{15}$ These early systems were designed for specific types of measurements and, therefore, lacked the flexibility and control over liquid properties necessary for a systematic study of acoustic cavitation phenomena. Our goal is to develop an apparatus that (i) facilitates control of liquid properties such as temperature, dissolved gas content, surface tension, particulate contamination, ionic content, and chemical impurity; (ii) is flexible enough to potentially accommodate a variety of cavitation generation systems (spherical resonators, pulsed transducers, etc.) and detection schemes (sonoluminescence, sound emission, etc.); and (iii) can produce large quantities of reproducible threshold data in an automated fashion. We now briefly describe the specific details of the experimental apparatus. For a detailed description, see Ref. 16. 


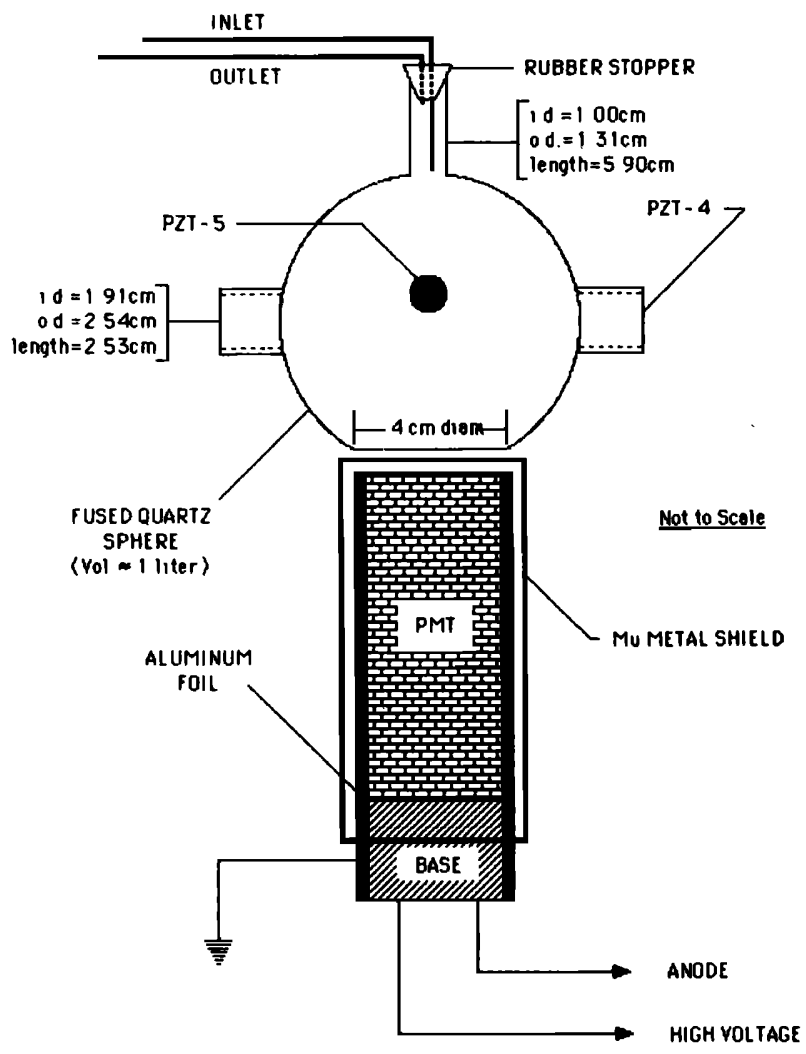

FIG. 1. Diagram of the resonance cell-photomultiplier tube assembly.

\section{A. Resonance sphere and PMT assembly}

The acoustic standing wave is generated by a 1-1, fused quartz, spherical resonator with dimensions as outlined in Fig. 1. The sphere is driven by two PZT-4 cylindrical transducers (resonance frequency $\approx 50 \mathrm{kHz}$ ) epoxied on the sides as shown. Also epoxied to the cell are a miniature PZT-5 pill transducer (to monitor the sound field) and a linear thermistor. The fill tubes ( $\approx 3.5-\mathrm{mm}$ o.d.) are supported by a twohole rubber stopper, with the inlet tube extending down to the mouth of the sphere to facilitate adequate mixing when circulating the liquid. Facing the flat is an RCA 6342A photomultiplier tube shielded by a combination of a Mu metal jacket and a grounded aluminum foil wrapping. The entire cell/PMT assembly is housed in a "light tight" plywood enclosure.

Due to the extreme complexity of the resonant modes of the cell, we require a "roughly spherical" mode with a pressure maximum at the center (to eliminate boundary effects). Empirical observations of gassy water (stable cavitation) indicate that the best mode, both in terms of spatial configuration and $Q$, occurs at a frequency of $61.725 \mathrm{kHz}$. Repeated visual observations with degassed water show that transient cavities evolve in the center and nowhere else. This "cavitation zone" is about $1.5 \mathrm{~cm}$ in diameter.

The spherical flask configuration, which was suggested by Blake ${ }^{15}$ and utilized by many others, ${ }^{9,17-19}$ is simple to construct, optically visible, easily cleaned, and has few contaminating interfaces. However, our resonator is limited to a maximum acoustic pressure of about 15 bars.

\section{B. Fluld management system}

Figure 2 illustrates the fluid management system devised to control the liquid properties discussed previously. The system is closed, as suggested by Greenspan and Tschiegg, ${ }^{20}$ who pointed out that the liquid must be continuously circulated to achieve adequate cleanliness. A magnetic drive metering pump circulates the entire bulk of the fluid $(\approx 2$ liters) in about $5 \mathrm{~min}$. All fluid handling surfaces are

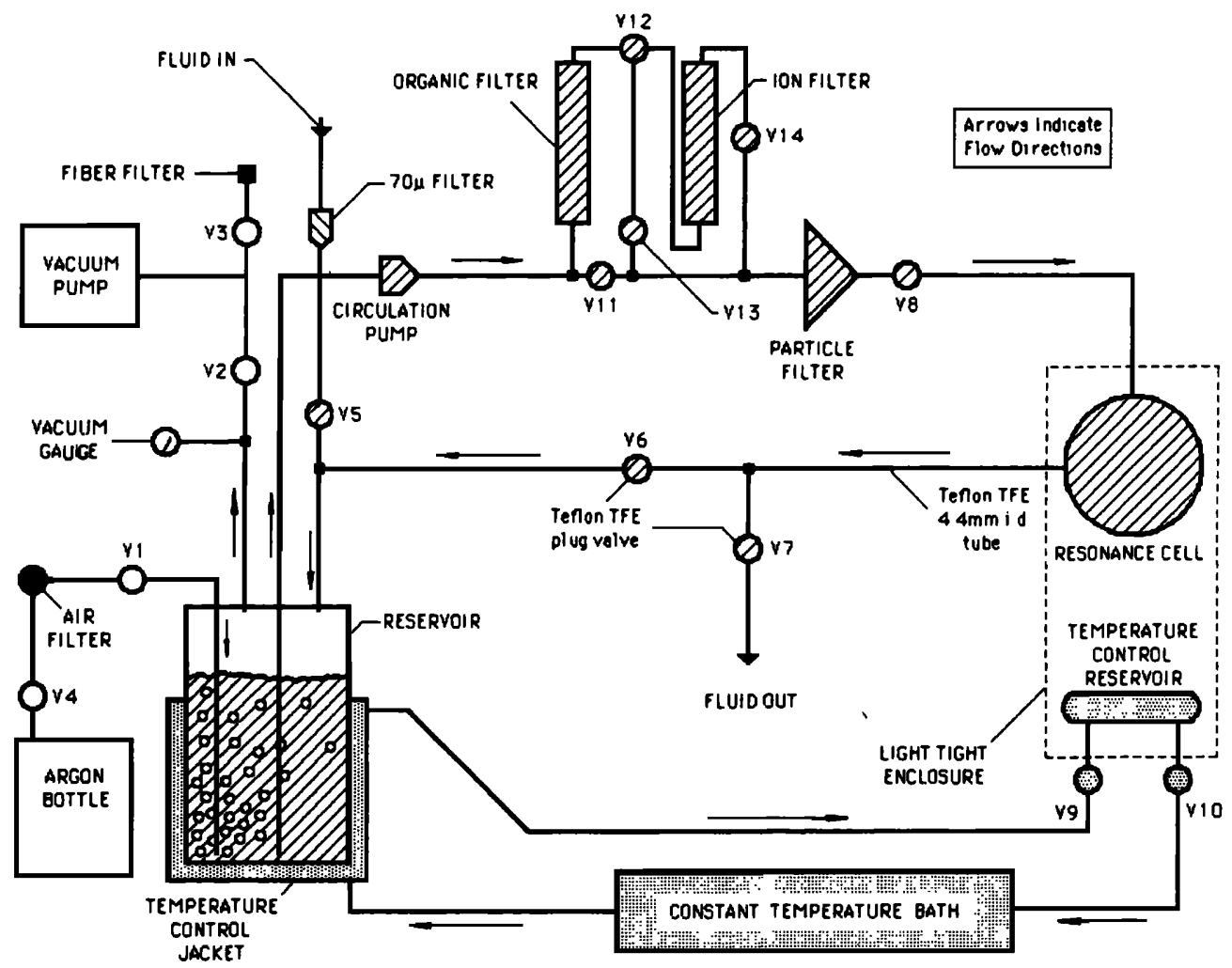

FIG. 2. Block diagram of the fluid management system. All fluid handling materials are tefion, glass, or stainless steel. V5 and V7 (which are plug valves) are normally closed, except when adding or removing fluid from the system. 
either glass, teflon, or stainless steel.

We control the gas concentration by simultaneously evacuating the resevoir to a desired pressure, bubbling argon (which gives a more intense sonoluminescence response than air ${ }^{21}$ ), and circulating the liquid. The resulting "dissolved argon concentration" (the present quantity of argon compared to that at saturation) is measured with a volumetric Van Slyke apparatus. This ratio is reproducible to within $2 \%$ and variable from about $30 \%$ to $100 \%$ of saturation.

Temperature control is provided by a constant temperature jacket surrounding the fluid reservoir and a 4-1 constant temperature reservoir within the light tight box. The temperature at the wall of the sphere can be reproducibly set and maintained to within $1^{\circ} \mathrm{C}$.

The size of the solid particulate matter in the liquid is controlled via careful and extensive filtration through frequently replaced Millipore filters (visual inspections reveal little or no observable deposits on the surface during the latter stages of filtration). Prior to each experiment, the sample undergoes simultaneous filtration and "argonation" for $45 \mathrm{~min}$ through filters that remain as clean as possible. During this time the bulk of the fluid makes at least nine passes through the system; thus the particle size distribution has an upper bound that is approximately equal to, or slightly less than, the filter pore size. Since the organic skins described by Yount are very tough, his nuclei may be regarded as "particles" and are filterable (as are dirt particles). We therefore assume that filtration also establishes a rough upper bound to the nuclei size distribution in the liquid.

Most chemical impurities are removed by passing the liquid through Sybron-Barnstead organic and ionic removal columns. This system lowers the content of high molecular weight organics to a few parts per million and increases the resistivity to $1 \mathrm{M} \Omega / \mathrm{cm}$. Any degree of chemical impurity or ionic concentration is then achieved by adding to the reservoir controlled quantities of a desired chemical or salt.

\section{Cavitation generation system}

The essential nature of the cavitation generating system is that the sound field at the center of the resonance cell is increased linearly with time, at reproducible rates, until cavitation occurs. The system then automatically resets into a predetermined quiescent state (discussed later) for a fixed period of time, after which the timer resets the ramp. The actual sequence of events that constitute a measurement will be given later.

Figure 3 shows a block diagram of the cavitation generation apparatus. The heart of the system is a ramp generator (our design) that drives both the amplitude modulation input of a function generator and a strip chart recorder. The dc voltage level of the strip chart record is linearly related to the peak-to-peak output amplitude of the function generator. This signal is amplified and delivered to the PZT-4 driving transducers mounted to the sides of the sphere. The resulting acoustic standing wave is "monitored" by a PZT-5 pill transducer whose output is fed into an oscilloscope and a digital resonance tracking circuit (our design). This device maximizes the rms output of the cell by adjusting the frequency of the function generator (via the VCO input) to track the approximate resonance. Acoustic pressure measurements with a probe hydrophone have shown that the resonance frequencies measured at the center of the sphere and at the pill agree to within the uncertainty of the tracking circuit, which is less than $\pm 5 \mathrm{~Hz}$ at $61.725 \mathrm{kHz}$. During a typical experiment,

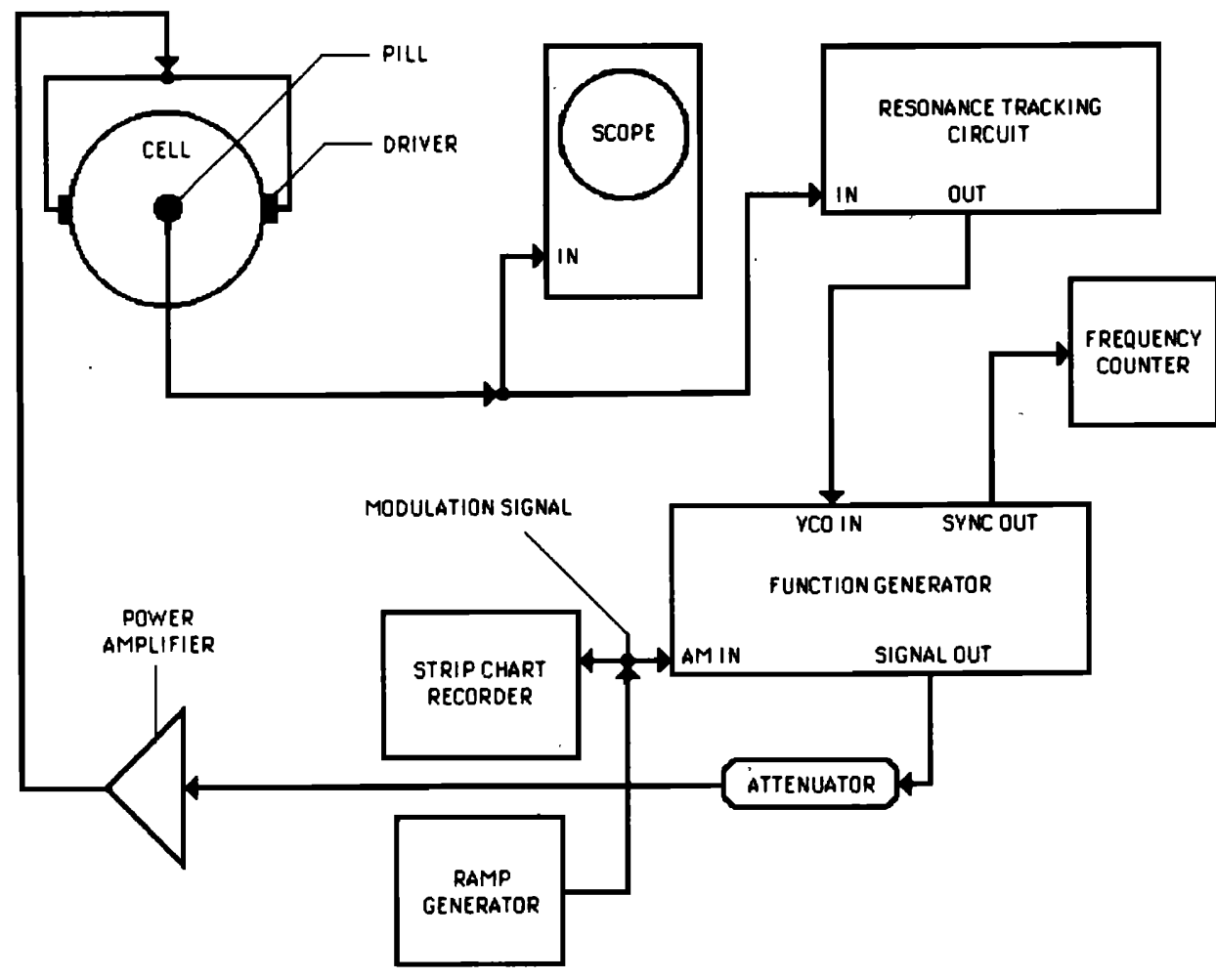

FIG. 3. Block diagram of the cavitation generation system. 
the resonance frequency will increase by as much as $20 \mathrm{~Hz}$, primarily due to heating.

The system is calibrated by determining the acoustic pressure amplitude in the center of the cell $P_{a}$ as a function of the strip chart voltage $V_{s}$. Using a calibrated probe hydrophone (calibrated after a technique suggested by Gould ${ }^{22}$ and discussed in detail by Crum and Eller ${ }^{23}$ ), we monitor the sound field at the secondary maxima (to avoid cavitation at the probe tip) and employ the experimentally measured relationship between the primary and secondary pressure amplitudes (found to be linear) to determine $P_{a}$ vs $V_{s}$. The resulting calibration is given by the relationship $\boldsymbol{P}_{a}$ $=2.87 V_{s}+0.91$, where $V_{s}$ is in volts and $P_{a}$ in bars. Once calibrated, the strip chart recorder automatically keeps a running record of the pressure amplitude in the cell.

\section{Cavitation detection system}

Figure 4 shows the cavitation detection apparatus together with the gating, timing, and triggering circuitry. Sonoluminescence generated by a transient cavity is detected by the photomultiplier tube and the resulting pulse from the PMT base is preamplified and fed into a pulse height discriminator. The discriminator level determines the sensitivity of the apparatus and is set to $600 \mathrm{mV}$, which is well above the electrical background level $(\approx 150 \mathrm{mV})$ but below the typical sonoluminescence pulse height $(\approx 1.0 \mathrm{~V})$. After discrimination, the signal passes through a "noise gating" network (described below) followed by a linear gate and results in the

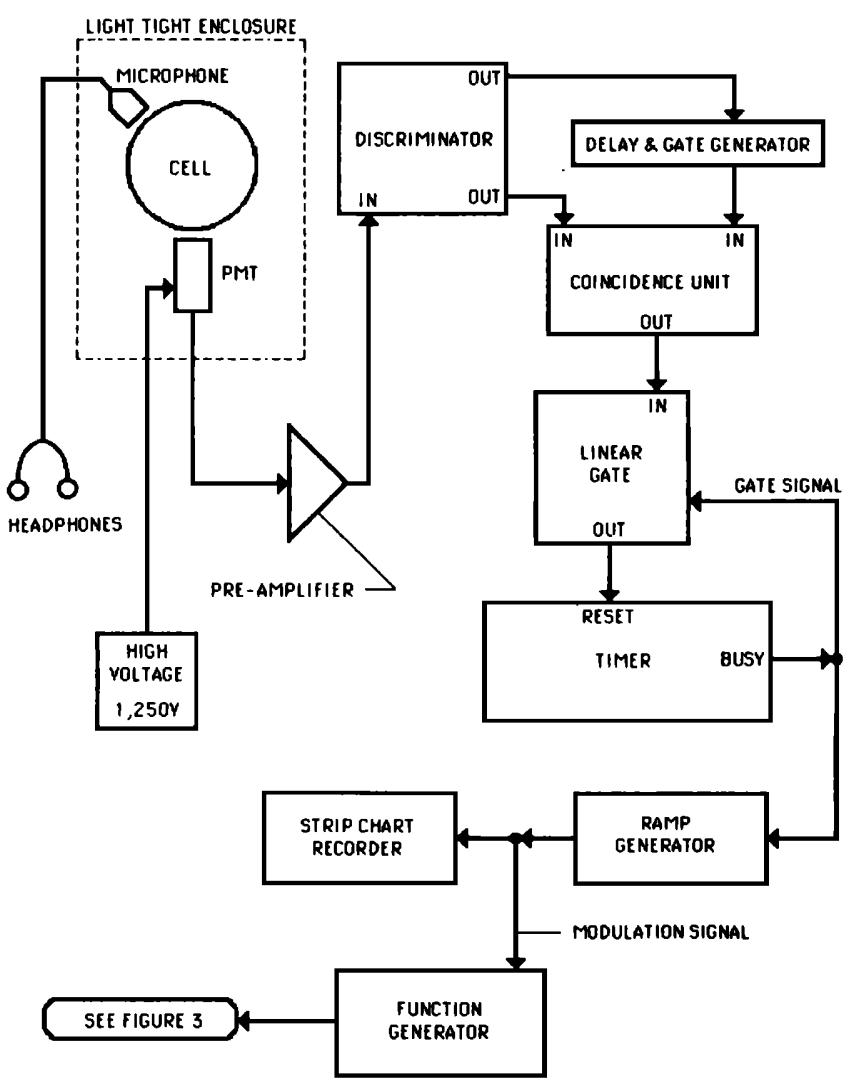

FIG. 4. Block diagram of the cavitation detection system. "trigger" pulse that resets the system. A "system reset" refers to an immediate reduction in the sound pressure amplitude to a quiescent level $(0.3 \mathrm{bar})$ for a predetermined length of time.

During the quiescent interval, both the linear gate and the ramp generator are disabled via the "busy" output of the timer. At the end of the timing sequence the busy signal goes low, which opens the linear gate and engages the ramp generator. The ramp proceeds until a transient cavitation event generates a pair of light pulses which, provided they survive discrimination and "noise" gating, reset the timer and the whole process repeats itself.

In this configuration, there exists a background "light noise" level of about five pulses per minute, probably due to Cerenkov radiation. The linear gate prevents any stray pulses from resetting the timer before the quiescent period is over. We desire a slow ramp rate, however; with noise signals occurring about every $12 \mathrm{~s}$, we cannot wait too long for cavitation to occur. Pulse height discrimination is ineffective since the brightest noise pulses are more intense than the weakest sonoluminescence events. In this system, transient collapses almost always occur in bunches, with some events producing hundreds of pulses within milliseconds. This fact inspired a "noise gating" scheme where one output of the pulse height discriminator generates a delayed, 100-ms-long gate signal. The gate is fed, along with the second discriminator output, into a coincidence unit. The coincidence output, which is enabled by the occurrence of two discriminator pulses separated by no more than $100 \mathrm{~ms}$, provides the trigger signal that resets the system. This technique allows us to halve the discriminator level, while increasing the noise immunity by a factor of seven.

\section{E. Experimental procedure}

Prior to an experimental run, the physical properties of the liquid are brought to a desired level, after which the sample sits undisturbed for at least $1 \mathrm{~h}$. Data acquisition is automatic; the system cycles between a 200-s quiescent state $\left(P_{a}=0.3\right.$ bar $)$ and a 0.2 -bar-per-second ramp to the threshold until the operator disengages the apparatus. The quiescent period provides time for dissolution of any nonstabilized microbubbles that may result from a violently collapsing bubble (or group of bubbles). Measurements indicate that a significant reduction in this quiescent time (e.g., 60 s) does not affect the mean experimental threshold; the standard deviation, however, increases. A typical data run yields about 30 measurements taken over a 1.6-h period.

In two separate experiments, we measured the dependence of the transient cavitation threshold on nuclei size and dissolved ion concentration. The approximate upper bound to the nuclei size distribution was established by cautious and extensive filtration. The ion concentration was varied by first deionizing the water and then adding measured amounts of potassium iodide or sodium iodide. For both experiments, the test fluid was triply distilled water at a temperature of $23^{\circ} \pm 0.5^{\circ} \mathrm{C}$ and a surface tension of $71.3 \pm 0.2$ $\mathrm{dyn} / \mathrm{cm}$. The dissolved argon concentration was $50 \% \pm 2 \%$ 


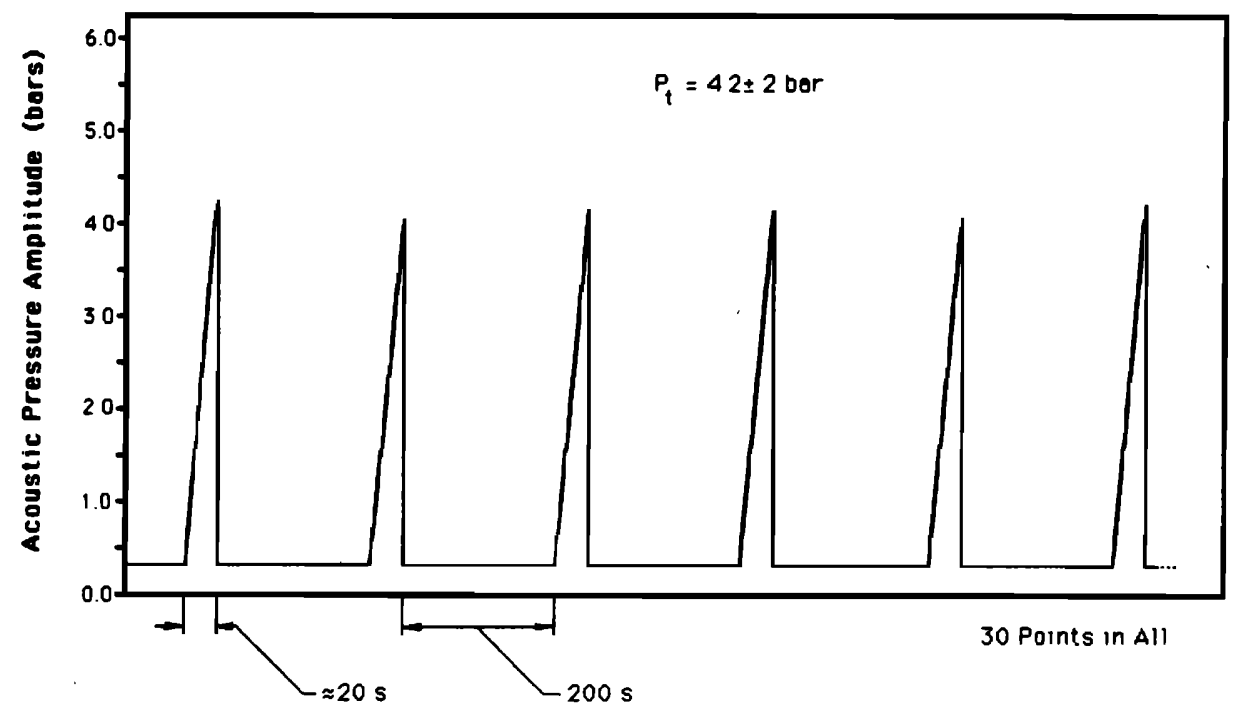

FIG. 5. Reproduction of a strip chart record demonstrating the technique for cavitation threshold measurements and the reproducibility of the data. The driving frequency was $61.725 \mathrm{kHz}$. The liquid was distilled water circulated through a $20-\mu \mathrm{m}$ filter, at a dissolved gas concentration of $50 \%$ of saturation, a surface tension of $71.3 \mathrm{dyn} / \mathrm{cm}$, and a temperature of $23.0^{\circ} \mathrm{C}$.

of saturation for the filter experiment and $30 \% \pm 2 \%$ of saturation for the ion experiment.

\section{RESULTS AND DISCUSSION}

Figure 5 is a partial reproduction of the strip record for a typical set of data. The sample was distilled water, filtered only to remove large particles $(20-\mu \mathrm{m}$ filter pore diameter) and degassed to $50 \%$ of saturation. The entire record contains 30 data points and the resulting cavitation threshold $\left(P_{t}\right)$ is $4.2 \pm 0.2$ bars, which is in quantitative agreement with previous work. ${ }^{19,17,24}$ Figure 5 is representative of all 30 points and serves to illustrate the high precision of the apparatus; a $4.8 \%$ relative deviation in cavitation threshold data is, to our knowledge, unprecedented in the literature. Such precision means that small changes in the threshold (caused by changes in the liquid) are distinguishable. The measured thresholds are repeatable (within a standard deviation) from one day to the next and between identically prepared samples of water from the same source.

A second example is given in Fig. 6. The liquid here is filtered to $5 \mu \mathrm{m}$ (particle diameter) and the horizontal lines

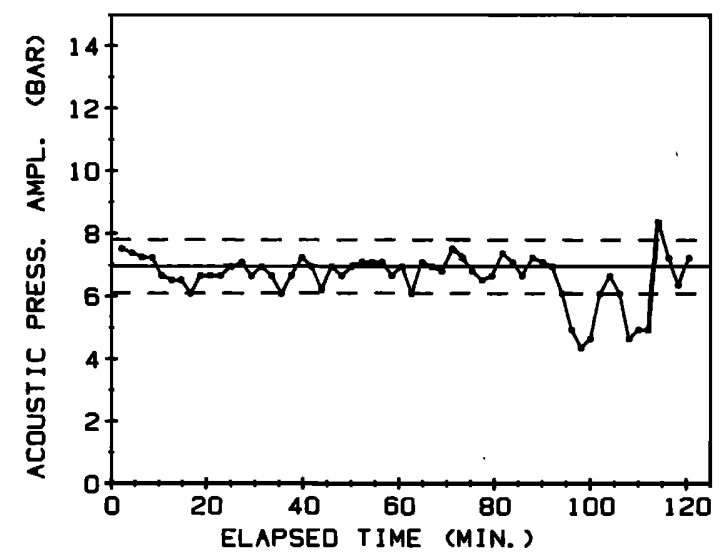

FIG. 6. Measurements of the cavitation threshold as a function of time Aside from the filter size ( $5 \mu \mathrm{m}$ ), the same conditions apply as in Fig. 5. define a width equal to \pm 3 standard deviations of the cavitation threshold. The threshold is essentially constant for about $90 \mathrm{~min}$, after which significant "oscillations" are observed. Having ruled out systematic experimental error, we find this behavior inexplicable. Perhaps "aging" the liquid results in coalescence of nuclei near the center of the cell, thereby reducing the threshold. This reproducible effect is more evident when the quiescent time interval is short $(120 \mathrm{~s}$ for this data) and when the sample is more extensively filtered.

\section{A. Threshold versus filter size}

The above data support the concept of a reasonably stable, well-defined cavitation threshold. Assuming the threshold depends on nuclei size, this suggests the existence of a sharp "edge" in the nuclei size distribution. Previous experiments with ordinary unfiltered tap water ${ }^{9.24}$ exhibit a quantitatively similar, well-defined threshold. Therefore, it is unlikely that filtration through the $20-\mu \mathrm{m}$ filter significantly truncated the nuclei size distribution. Plotted in Fig. 7 is $P_{t}$,

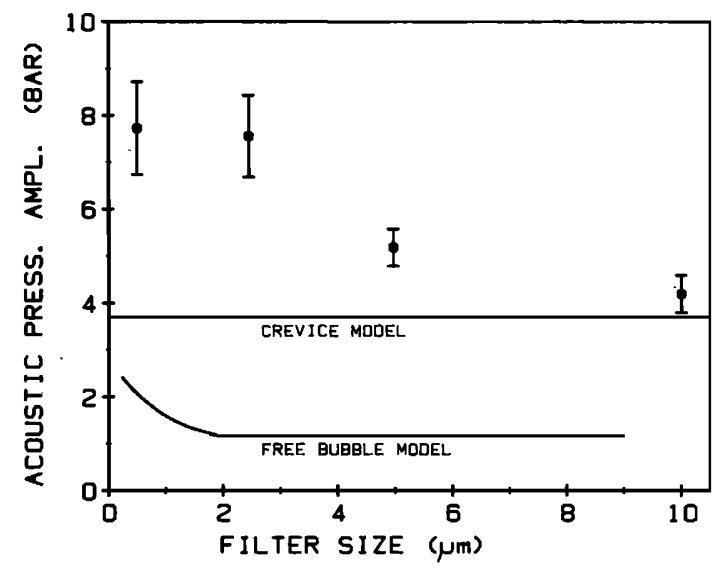

FIG. 7. Measurements of the acoustic cavitation threshold as a function of the filter size (pore radius) for distilled water. The error bars refer to \pm 1 standard deviation. Apart from the filter size, the same conditions apply as in Fig. 5. The two theoretical curves refer to predictions based on conventional models of the cavitation nucleus. 
the threshold pressure amplitude, versus filter size (pore $r a$ dius) ranging from 10 to $0.5 \mu \mathrm{m}$. Each data point is the arithmetic mean and standard deviation (error bars) of 30 measurements taken on two different occasions with the same liquid. Both the mean threshold and the standard deviation increase with decreasing filter size. Note that as the nuclei are depleted, there is a growing probability that a lowthreshold nucleus will not be in the cavitation zone when the threshold pressure is reached. This effect, which varies inversely with the ramp rate, increases both the mean measured threshold and the standard deviation. Observations indicate, however, that only at large ramp rates (greater than about $1 \mathrm{bar} / \mathrm{s}$ ) is the error greater than a few standard deviations, not enough to explain the trend in the data.

Also shown in Fig. 7 are the predicted dependences of $P_{t}$ versus nuclei radius for a crevice model, using an explicit expression of Crum, ${ }^{10,24}$ and for a free bubble model that is essentially the threshold given by Blake $e^{25}$ and modified by Roy ${ }^{16}$ to include some bubble dynamic effects obtained by Apfel. ${ }^{26}$ The crevice model exhibits quantitative agreement at large filter sizes but fails to explain the increase in threshold for smaller nuclei. The free bubble model predicts a rise in the threshold at small filter sizes; however, there is poor quantitative agreement between theory and experiment at all filter sizes. A detailed discussion of the possible theoretical reasons for these discrepancies is beyond the scope of this paper and will be presented in a later report.

\section{B. Threshold versus lon concentration}

Akulichev $^{2}$ has suggested (on the basis of his measurements which show an inverse dependence of the threshold on the concentration of such salts as $\mathrm{KI}$ and $\mathrm{KBr}$ ) that a free bubble can be stabilized by hydrophobic ions congregating on or near its surface. Syrotyuk, ${ }^{27}$ however, observed essentially no variation in threshold with electrical conductivity. Using the ion removal column, we have somewhat verified and extended upon the measurements of Akulichev.

We measured the cavitation threshold in filtered water (7- $\mu \mathrm{m}$ particle diameter) as a function of dissolved $\mathrm{KI}$ concentration over a range from $10^{-2} \mathrm{mmole} / 1$ to $10 \mathrm{mmole} / \mathrm{l}$, which is about two orders of magnitude greater than Akulichev's range. The measured thresholds are plotted in Fig. 8. They exhibit a complex dependence on ion concentration, rising to a peak value near $1 \mathrm{mmole} / 1$ and then decreasing with increasing concentration. Since Akulichev's measurements were made at a dissolved gas concentration of $13 \%$ and there is some uncertainty about his initial ion concentration, direct comparison of the two results is diffucult. Plotted as a dashed line, however, is an estimate of his values; the qualitative agreement is evident. A detailed discussion of these results awaits a later report.

\section{Sonoluminescence versus sound emission}

To this point, we have discussed only transient cavitation thresholds determined via light emission. This system is also equipped to employ audible sound emission as a cavitation indicator, where the operator "listens in" on headphones (see Fig. 4) and manually activates a system reset

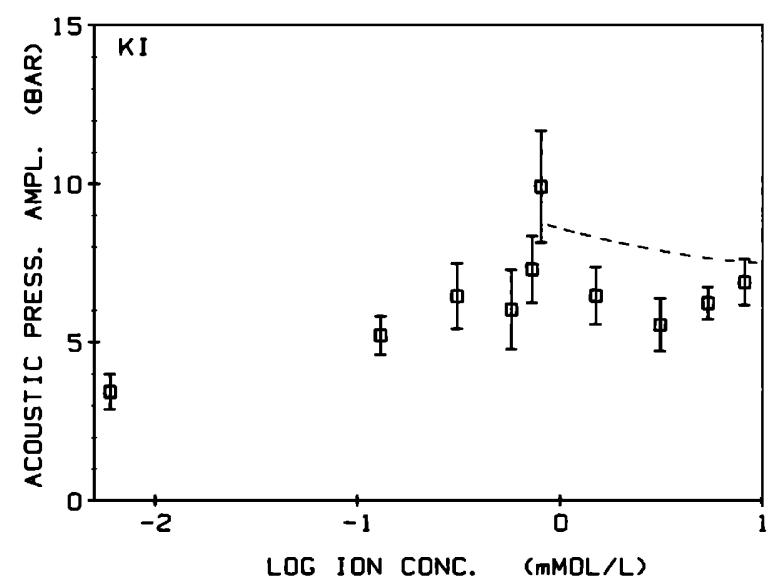

FIG. 8. Measurements of the acoustic cavitation threshold of distilled water as a function of the concentration of dissolved KI. Aside from the filter size $(7 \mu \mathrm{m})$ and the gas concentration (30\%), the same conditions apply as in Fig. 5. The dashed line represents an estimate of Akulichev's ${ }^{2}$ data.

when a click or pop is heard. Figure 9 shows data for which both the audible and the sonoluminescence thresholds were simultaneously measured as a function of $\mathrm{NaI}$ concentration in filtered $(7 \mu \mathrm{m})$ water. Both light and sound generation are characteristics of transient cavitation, yet the threshold for audible sound emission is always less than or equal to that for light emission. Observations made while compiling the data of Fig. 7 indicate that as the nuclei size gets smaller, the thresholds for sound emission and sonoluminescence diverge, the latter having the higher threshold. Apfel ${ }^{26}$ (drawing on the work of Neppiras and Noltingk) defines a transient cavity as one which satisfies the criterion $R_{m} /$ $R_{0}=2.3$, where $R_{0}$ is the initial radius of the bubble (nuclei size) and $R_{m}$ is the maximum radius it grows to. This corresponds to the point where the collapse velocity equals the speed of sound in the liquid, in the incompressible assumption, and establishes a threshold for sound emission. On the other hand, Neppiras ${ }^{28}$ has shown that the temperature generated by a collapsing bubble is proportional to $R_{m}$ which, at the threshold for sound emission, decreases with decreasing

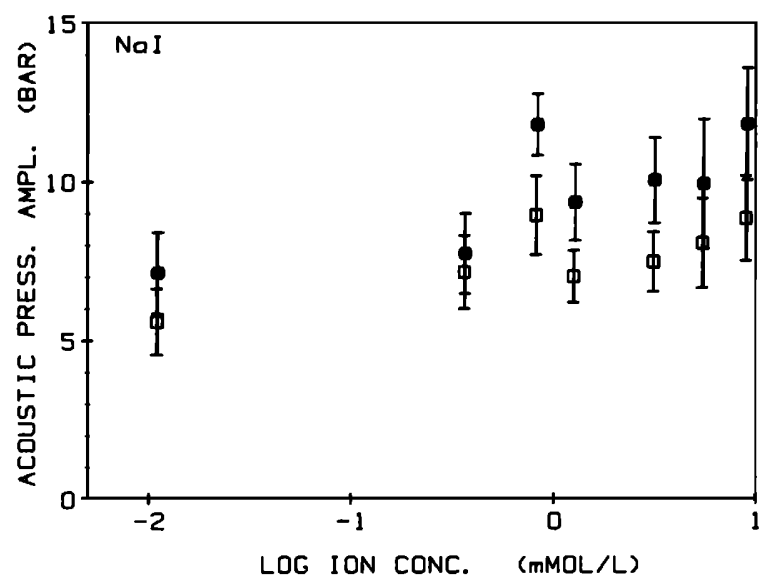

FIG. 9. Measurements of the acoustic cavitation threshold of distilled water as a function of dissolved $\mathrm{NaI}$ concentration. The open squares represent measured thresholds using an audible click as a criterion; the solid squares are the values using sonoluminescence. 
nuclei size. Thus an increase in the sonoluminescence threshold relative to the sound emission threshold for smaller filter sizes seems plausible.

Although there are many subtleties here (such as the choice of discriminator setting), it is apparent that sound and light emission indicate thresholds for two different types of phenomena associated with transient cavitation. If one desires a threshold for "violent" cavitation, then sonoluminescence is a fitting criterion since the violence of a transient event is linked primarily to $R_{m}$, which corresponds to the energy stored in the liquid. Thus light emission may serve as an ideal indicator of what Apfel ${ }^{26}$ calls the "threshold for transient-violent cavitation," an idea that warrants further investigation.

\section{Summary and conclusions}

We have developed an apparatus that performs acoustic cavitation threshold measurements in an automated, precise, and reproducible manner. The present system relies primarily on a detection scheme (sonoluminescence) that is indicative of cavitation violence. Therefore, the threshold can be vigorously specified. Because the operator exercises complete control over the liquid properties, the influence of many physical parameters on the threshold can be selectively examined. The system is versatile, permitting detection schemes based on both light and sound emission. Also, the apparatus can accommodate, with straightforward modifications, other cavitation generating schemes such as cylindrical resonators, focused transducers, etc. (we are currently running experiments with focused transducers driven by tone bursts).

We found the transient cavitation threshold of distilled, filtered water to be well defined, reproducible, and reasonably stable. However, finely filtered samples exhibit, after about 90 min, periodic fluctuations in cavitation thresholds as a function of time. These results appear anomalous.

Measurements of the threshold as a function of filter size (pore diameter) indicate a gradual rise from about 4 bars for a filter size of $20 \mu \mathrm{m}$ to nearly 8 bars at a filter size of $1 \mu \mathrm{m}$. These results are as yet inexplicable on the basis of conventional nucleation models.

Measurements of the cavitation threshold for aqueous solutions of certain halide salts indicate a dependence on the dissolved ion concentration. A reduction in the threshold over that of ordinary distilled water is observed for highmolar concentrations, which verifies the earlier results of Akulichev. ${ }^{2}$ However, we also observe a threshold reduction for low-molar concentrations, where dissolved ions are removed from ordinary distilled water. Existing nucleation models cannot account for the latter result.

Transient cavitation thresholds measured using an audible noise criterion are less than or equal to thresholds based on sonoluminescent emission. This difference, which does not appear to be entirely due to the detection levels required, involves the details of bubble dynamics.

\section{ACKNOWLEDGMENTS}

We wish to acknowledge the financial support of the National Science Foundation, the Office of Naval Research, and the Department of Health and Human Services.

'E. N. Harvey, D. K. Barnes, W. D. McElroy, A. H. Whiteley, D. C. Pease, and K. W. Cooper, J. Cell Comp. Physiol. 24, 1-22 (1944).

${ }^{2}$ V. A. Akulichev, Sov. Phys. Acoust. 12, 144-149 (1966).

${ }^{3}$ F. E. Fox and K. F. Herzfeld, J. Acoust. Soc. Am. 26, 985-989 (1954).

${ }^{4}$ D. E. Yount, J. Acoust. Soc. Am. 65, 1429-1439 (1979).

${ }^{5}$ J. W. Holl, J. Basic Eng. (TRANS ASME) Series D 92, 681-688 (1970).

${ }^{6}$ R. E. Apfel, "Acoustic Cavitation," in Methods of Experimental Physics, edited by P. Edmonds (Academic, New York, 1981), Vol. 19, p. 355.

${ }^{7}$ L. A. Crum, Appl. Sci. Res. 38, 101-115 (1982).

${ }^{8}$ D. E. Yount, T. D. Kunkle, J. S. D'Arrigo, F. W. Ingle, C. M. Yeung, and

E. L. Beckman, Aviat. Space Environ. Med. 48, 185-191 (1977).

${ }^{9} M$. Strasberg, J. Acoust. Soc. Am. 31, 163-176 (1959).

${ }^{10}$ L. A. Crum, Nature 278, 148-149 (1979).

"'R. E. Apfel, J. Acoust. Soc. Am. 48, 1179 (1970).

${ }^{12}$ G. ter Haar and S. Daniels, Phys. Med. Biol. 26, 1145-1149 (1981).

${ }^{13}$ F. G. Sommer and D. Pounds, Med. Phys. 9, 1-6 (1982).

${ }^{14}$ E. A. Neppiras and W. T. Coakley, J. Sound Vib. 45, 341-373 (1970).

${ }^{15}$ F. G. Blake, Jr., "The Tensile Strength of Liquids, a Review of the Literature," Tech. Memo No. 9, Acoustics Research Laboratory, Harvard University (1949).

${ }^{16}$ R. A. Roy, "The Role of Nuclei Size in Acoustic Cavitation Threshold Measurements," M.S. thesis, University of Mississippi (1984).

${ }^{17}$ W. J. Galloway, J. Acoust. Soc. Am. 26, 849-855 (1954).

${ }^{18} \mathrm{~J}$. Barger, "Thresholds of Acoustic Cavitation in Water," Tech. Memo No. 57, Acoustics Research Laboratory, Harvard University (1964).

${ }^{19}$ P. W. Vaughan, E. Graham, and S. Leeman, Appl. Sci. Res. 38, 45-54 (1982).

${ }^{20} \mathrm{M}$. Greenspan and C. E. Tschiegg, J. Res. Nat. Bur. Stand. 71C, 299-312 (1967).

${ }^{21}$ R. O. Prudhomme and T. Guilmart, J. Chem. Phys. 54, 336 (1957).

${ }^{22}$ R. K. Gould, J. Acoust. Soc. Am. 43, 1185 (1968).

${ }^{23}$ L. A. Crum and A. I. Eller, "The Motion of Bubbles in a Stationary Sound Wave," Tech. Memo. No. 61, Acoustics Research Laboratory, Harvard University (1969).

${ }^{24}$ L. A. Crum, "Acoustic Cavitation Thresholds in Water," in Cavitation and Inhomogeneities in Underwater Acoustics, edited by W. Lauterborn (Springer, New York, 1980), p. 84.

${ }^{25}$ F. G. Blake, Jr., "The Onset of Cavitation in Liquids," Tech. Memo. No. 12, Acoustics Research Laboratory, Harvard University (1949).

${ }^{26}$ R. E. Apfel, J. Acoust. Soc. Am. 69, 1624-1630 (1981).

${ }^{27}$ M. G. Sirotyuk, Sov. Phys. Acoust. 16, 237-240 (1970).

${ }^{28}$ E. A. Neppiras, "Acoustic Cavitation," in Physics Reports (North-Holland, Amsterdam, 1980), Vol. 61. 